\title{
A Pathologist Goes to War: The Prior's Papers Paper II
}

Col E E Vella, MD, FRCPath, L/RAMC

Assistant Editor, Journal of the Royal Army Medical Corps

\section{Short Biography of A P Prior}

Major Allen Percival Prior, MB (Sydney), FRCPath was a resident pathologist in the Royal North Shore Hospital Sydney; the outbreak of the 1939-1945 War found him on his way to the UK to undertake a postgraduate course. He immediately volunteered for war service and was commissioned in the RAMC in October 1939, whereupon he was posted as RMO to the 106 Regt RHA, Middle East Theatre.

He soon became a graded specialist in pathology (? No 13 General Hospital, Suez) and subsequently a specialist pathologist in the rank of Major on the staff of No. 8 General Hospital, Alexandria.

In 1944 he was RHE back to UK, and in the closing months of the war he was in Germany (? No 9 British General Hospital) entering Belsen Concentration Camp as soon as this had been liberated by British troops.

He was demobilised in 1945 but decided to stay in UK and in 1947 was appointed county pathologist to the South Warwickshire Hospital Group.

.He died in Canberra, Australia on January 2nd, 1971 aged 62 , only one day after his premature retirement.

Amongst his documents there is a very thick file of Post Mortem Reports from MELF covering the deaths from natural and unnatural causes of personnel belonging to the various nationalities who were involved in that crucial theatre of operations; there are also Post Mortem Reports from the European theatre of war.

Apart from the expected causes of death, namely those due to injuries in the battle zones, there are also other reports, some of which are rather exotic findings, such as for example:

1. Lieut C I Pagirski, RAMC, attached 4 Libyan Arab Forces. Cause of death poisoning by opium derivatives potentiated by ethanol.

2. One of the shortest post mortem reports that I have seen, that of the unfortunate $\mathrm{L} / \mathrm{Cpl}$ Sephepha Ramat La Peng, 1914 Basuto Garrison Coy, who was decapitated by a steam locomotive, and

3. A still shorter and more succinct report, that on the body or rather more accurately on the human torso recovered from the sea..(Fig. 1).

Other causes of death attributed to non-traumatic aetiology include dysentery and typhoid, typhus and $\overrightarrow{\vec{\omega}}$ tuberculosis, diphtheria and lobar pneumonia, falciparumg malaria and hepatic amoebiasis, etc.

\section{SECTIONI}

Not so Elementary my dear Watson! Was it Suicide, Homicide or Accidental Death?

\section{Capt M P M, Royal Malta Artillery, 5/2 HAA}

On receipt the body was clad only in a blanket. It bore? a religious medallion.

The body showed post mortem hypostasis and was o bright cherry colour. The same bright colour was evide on the lips.

On opening the body the blood and colour of the internale organs appeared pink.

A small sample of post mortem blood was dilutedo progressively up to one part in several thousands. Tiss

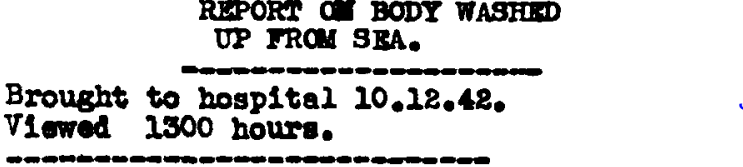

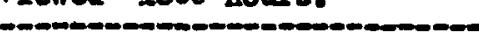

The lower portions of all four limbs and the head were mlsalns. The trunk showed slens of lwmeralon.

It was ocompletely unsidentirlable.

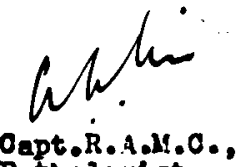

Pathologlet.

10.12.42. No.8.0eneral Hoopital.

Fig. 1 The shortest Post Mortem Report that I have seew. Assistant Editor. 
showed a characteristic cherry red colour. Normal control blood showed yellow in the same dilution.

Conclusion: In my opinion death was due to Carbon Monoxide poisoning.

\section{Unidentified body recovered from sea (near Ras-el-Tin).}

Greatcoat and trousers examined by SIB personnel. Body clothed in khaki shirt only. No identifying marks on shirt. Aged probably about 22 years. No scars.

External Examination: Diffuse red staining of face and scalp. Numerous superficial cuts on face. Skin of palms, wrists and soles of feet sodden and beginning to desquamate. Large number of linear pressure marks over arms, chest and legs. Nothing grasped in hands. Rigor mortis in jaw, trunk and legs, slight in shoulders, elbow and wrists, absent in neck.

Mouth contains small pieces of seaweed and debris. Frothy blood-stained fluid in trachea and bronchi, with fine sand as far as bifurcation of trachea. Lung voluminous and slightly waterlogged, with a few emphysematous bullae at edges. Heart contracted, especially on left side, right contains fluid blood.

\section{Cause of Death: Drowning}

Conclusion: I should estimate that the body has been in the water about 12 hours - possibly more - say up to 18 hours. This would place time of immersion between 2200 hours on 2.1.42 and 0400 hours on 3.1.42.

It is not possible to give an opinion as to time of death; obviously this might be considerably later than time of immersion.

Chemical examination of blood and the fluid from pleural cavity confirm that death was due to drowning. Blood chlorides $0.57 \%$ Hydrothorax fluid $1.31 \%$.

\section{SSM D A K. 3/CLY, HQ Sqdn}

Chest: Dislocation of Sterno-clavicular joint. Fractures of first, second, third, fourth, fifth and sixth ribs on right side close to the sternum. On the left side fourth, fifth and sixth ribs were broken anteriorly. Pleurae were torn, haemothorax present on both sides.

Abdomen: Multiple ruptures of liver. The bladder was halfway to the umbilicus.

Skull: Large depressed fracture on the right side.

Conclusion: Death was in my opinion due to multiple injuries which were not inconsistent with those sustained by a fall from a height.

\section{GNR F D, RA.241/61 AT Regt.}

There was a fracture of the skull present in the right temporo-parietal area; loss of bone substance for an area of $2.0 \mathrm{cms}$ by $1.0 \mathrm{~cm}$ of the upper and posterior part of the wing of the temporal bone and the lower lateral part of the parietal. Its edges shelved from without. Subsidiary fracture lines ran from this in various directions. One ran forward and to the left across the vault. One run forwards and then laterally to the middle cranial fossa, and two ranz backwards across the occipital bone. Cerebral substance $\stackrel{\mathbb{Q}}{\varrho}$ bulged through the hole.

Conclusion: The character and nature of the skull woundoํำ and cerebral laceration must give rise to serious" consideration of wounding by a severe blow from a blunt instrument.

\section{L/CPL H C C, CMP}

Over the surface of the right mastoid was a gaping wound with inverted edges. There was some burning and singeings of the posterior edge of the lobe of the ear. Inferior to the $\overrightarrow{0}$ wound was an area of powder blackening. This was $1.5 \mathrm{cms}$ from the nearest portion of the wound.

There was a small rounded wound with everted edges ands no surrounding powder burns on a line $0.5 \mathrm{cms}$ medial tos a line drawn vertically from the outer canthus and $2 \mathrm{cms}$ below the orbital margin. There was some denuding of thew epithelium immediately adjacent to the wound.

A track existed between these wounds. The wounds were in the same vertical place when the body was viewed in tEic anatomical position. This track passed through the antrifno and above the roof of the mouth, anterior to the clinow process, through the body of the right temporal bone whenth is considerably disrupted.

Conclusion: Death was due to injuries received fronap firearm. From the nature of the wound edges of posterior wound and the presence of powder marking gitoo seems probable that this was the entrance wound. In whith ${ }^{+}$ case the bullet had been fired from a level just below that of the victim's eyes, from behind, and on the right; a atids further, that the range must have been short - not abovio two metres.

However in order to decide these matters a knowledge o $\mathbb{B}$ the weapon and the circumstances would be necessary. $\overrightarrow{\vec{\sigma}}$

Assuming the above direction, it would be possible for 3 the wound to have been self inflicted. If the direction is reversed it would not be possible unless the victim was lef? handed. One can only regard the latter possibility aş extremely remote.

Further Report: To Assistant Provost Marshal Alexandria Area. Further to my autopsy report on L/Cpb H C C CMP and having viewed the scene of the shooting seen the firearm and the ammunition in question and having consulted the Medico-Legal Expert, I find mysel $B$ able to make the following additions:-

1. The firearm must have fulfilled certain conditions. In addition to the safety catch being in the appropriate position, the cocking arrangement being right and the trigger pulled:

(a) it must have been held steady in a horizontas plane.

(b) the muzzle of the firearm must have been at a distance of approximately $30 \mathrm{cms}$ from the entrance wound. The probable inside limit of 
distance is about $15 \mathrm{cms}$ and the outside limit $70 \mathrm{cms}$.

(c) The missile travelled from the right posterior aspect and emerged in the left anterior.

2. From the height of the victim and from the height of the bullet mark on the wall at the scene and the direction of the track, it is reasonable to conclude that both the victim and assailant were standing up and were erect.

3. Had the shot been fired during a struggle, as alleged, the victim's head must have been turned hard to the left whilst his right hand was well behind his right shoulder. His head could have in no way been flexed.

To summarise: The victim was killed by a bullet from a short range. This range was about $30 \mathrm{cms}$, the direction was from behind the right side of his head travelling towards the left front.

In order for this accident to have occurred in a manner described in the accused's statement the position of the victim - particularly as to his head - must have been such as I think any reasonable man would consider to be abnormal. One can say that it is possible for the accident to have occurred as described, but again I am of opinion that any reasonable man would consider this as unlikely.

\section{PO/X104094 Marine H S, MNBDO att 73 HAA, RA}

An entrance wound was present within left infraclavicular area anteriorly, and an exit wound in the posterior aspect just to the left of the midline in the lower part of the thorax. The bullet had evidently traversed in its course the greater part of the heart.

There was an aperture in the shirt in that position which had covered the entrance wound. No evidence of powder burning or blackening was present.

The missile has evidently come from the superior aspect of the victim and on the left side.

Conclusion: It is unlikely to have been self inflicted.

\section{T/-701, DVR F R. 10MTSD, RASC}

On the second left intercostal space $8 \mathrm{cms}$ from the midline was a rounded hole with inverted edges. The hole measured $0.3 \mathrm{cms}$ in diameter. On the inferior aspect of this hole was an area of powder burning extending downwards from the hole for a distance of $0.4 \mathrm{~cm}$; its lateral extent was $1.2 \mathrm{cms}$.

From the hole atract ran medially and posteriorly, first in the intercostal muscles, then through the medial aspect of the left pleura and lung, thence into the mediastinum, through the arch of the aorta, out into the right pleural cavity damaging the medial border of the right lung and thence into the body of the sixth rib posteriorly.

A bullet was discovered lying in the muscles of the posterior aspect of the sixth rib, pointing downwards and slightly laterally.

Conclusion: The track formed by the bullet forms an angle of approximately 45 degrees, with the plane vertical to the body and went medially and posteriorly and very slightly inferiorly. It would appear that the missile travelled from the left front aspect of the victim.

Without a more intimate knowledge of the habitus of the victim and an inspection of the clothing of the upper part of the body it is not possible to dogmatise as to whether this $\underset{\vec{D}}{\vec{D}}$ may have been self inflicted or not.

At the moment it seems possible but somewhat $\frac{\bar{\sigma}}{\bar{D}}$ improbable.

\section{SGT R J A , SAC MP, 16 Area}

No clinical information to the Pathologist.

There was a wound of the right temple $2 \mathrm{cms}$ superior to $\overrightarrow{\vec{\omega}}$ the posterior end of the zygoma. It had a central punched- $\stackrel{S}{S}$ in area but the surrounding tissue was slit in two directions so that it was roughly cruciate; surrounding this was an area of powder blackening some $4.0 \mathrm{cms}$ in diameter. The edges were inverted.

The left temporal area $3 \mathrm{cms}$ above the top of the middle part of the pinna was an irregular shaped wound whis measured $1 \mathrm{~cm}$ in greatest diameter. The edges wefo inverted.

A track existed between the entrance wound and the exi $\overrightarrow{7}$ wound on the left of the skull which passed through the substance of the brain at the level of the lateral ventricles with consequent haemorrhage within and without the dura

Conclusion: In my opinion death was due to injuries $\vec{\varphi}$ received from a firearm fired at close range.

This could have been self inflicted.

\section{CPL G W R, 4 Wks Gp, RE}

There was no evidence of disease. The soles of the feet $\frac{}{\Phi}$ were blackened apparently from walking barefoot.

The lower jaw and tissues of the lips were disrupted. An entrance wound with surrounding blackening and burning was present behind the right third molar; the track extended thence through the posterior pharyngeal muscles and emerged just anterior to the right auditory meatus.

Conclusion: Death was in my opinion due to injuries received from a firearm.

This was probably self inflicted.

\section{Result of Post Mortem Examination on \\ Unidentified Body washed up from the sea.}

This body is in an advanced stage of decomposition. Almost the entire superficial integument has been removed. 0

The whole of the skin and muscle of head and neck are gone.

It is not possible to identify either the arm of the Service or the person more accurately.

(Signed) A P PRIOR N Captain RAMC $N$ Pathologist

No 8 General Hospital. 


\section{SECTION II}

\section{Non Traumatic (sic) Post Mortems}

\section{PTE M, 1/Cameroon Highlanders}

The body was that of a well nourished male. There were numerous tattoo marks on the upper part of the trunk and arms. A ship and light-house (not in colours) was the central design on the chest. A bust of a Red Indian female was on either deltoid. There was no external evidence of injury. A pronounced odour of alcohol was present. This odour was even more marked on examining the various cavities.

Chest: Lungs were voluminous, and on section exuded quantities of blood and frothy fluid. The whole of the respiratory passages showed large amounts of frothy semitenacious pink stained mucus.

\section{Heart: NAD.}

Abdomen: The stomach was grossly dilated; it contained a large quantity of fluid and a small amount of semi-solid matter together with gas under tension. A clinical estimation of the size of the stomach was that it was about five to six times the size of normal.

Brain: The quantity of cerebro-spinal fluid was increased. The surface of the brain was of a pinkish colour. A moderate pressure cone was present. The fluid in the lateral ventricles was blood stained.

Conclusion: Death was due to Acute Alcoholic Poisoning.

\subsubsection{481 DESRAS SINGH, Punjabs}

Chest: Lungs were voluminous and tended to bulge outwards; on section they both oozed fairly copiously frothy blood stained fluid. There were haemorrhagic areas, from one to two cms in diameter, scattered throughout the parenchyma of the lung; these areas were oedematous on pressure but had not the appearance of infarcts.

Conclusion: In my opinion the appearances presented were consistent with Bomb Blast Injury.

Histology: Lung: Acute engorgement of bronchial vessels. Denudation of bronchial epithelium. Emphysema. Oedema. Patch haemorrhage into acini. Slight bronchopneumonia.

Opinion: Consistent with Blast but closely resembles Cordite Fume Poisoning.

(Signed) Pulvertaft Major RAMC DADP OC Central Pathological Laboratory c/o 15 (Scottish) General Hospital MEF.

\section{Pte W B, 4th East York Regt}

The body was that of a well proportioned young male. A number of superficial sores were present on the limbs. Most of these weredry but one on the right index was a little moist.
The penis had been subjected to operative interference where the dorsum of the prepuce had been split. The prepuce was swollen and oedematous and was dark ino

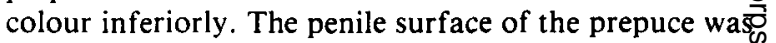
inflamed and showed membranous type of exudate. This: membrane was rather gelatinous in appearance ands extended over the whole penile surface of the prepuce.

Chest: No abnormality in upper respiratory tract. Lungso voluminous, crepitant, congested. Heart very much dilated.

Abdomen: Stomach, intestines, normal. Liver somewhates enlarged. Spleen congested. Kidney normal.

Brain: Normal.

Histology: Heart: Most of the fibres have lost their striations. In places the muscle fibres are replaced by structureless material. The bundles and the fibres are very well spaced out. In many places there is a not inconsiderable' emigration of leucocytes.

Prepuce: Sections from the prepuce show a large mass of inflammatory exudate, loss of epithelial structure, infiltration of leucocytes, early fibroblasts. Gram staindo sections show a variety of organisms on the surface and $\theta \mathrm{f}$ gram-positive bacilli in the deeper layers.

Bacteriology: cultures from the prepuce have giver⿸厂⿱二⿺卜丿) growth of Corynebacterium diptheriae.

Conclusion: In my opinion the macroscopic appearare at necropsy is consistent with a toxaemic death; it seems 50 most probable that this was due to the definitive organisin $s^{\star}$ above mentioned.

\subsubsection{GNR J H, RA, 145/42 LAA}

The dura mater was intact. The leptomeninges showed rather opaque but there was no tubercle formation or gross sign of infection. The surface of the brain was diffusely 3 reddened all over and the superficial vessels were congested $\bar{r}$ On section there were scattered throughout the cerebrump and cerebellum, both in the grey and white matter, a larges number of small haemorrhages. Nearly all of these could be called petechial.

There was no abnormality of the meninges of the cord In the cervical enlargement was an indefinite obscuring of the outlines of the anterior horns. The vessels were alF dilated and prominent. Quickly made paraffin sections from part of the cortex and of the cord show very much. enlarged vessels; polymorphonuclear leucocytes and smalp round cells together with some erythrocytes are to be found extravascularly in brain substance. No cuffing of vessels was found. Further histological investigations will be made $\frac{}{5}$ Conclusion: Whilst awaiting further evidence I am of the opinion that death was due to Polioencephalitis.

Histology: There is no evidence of diffuse encephalitis of other inflammatory change, there are however signs of fairly widespread damage to the brain in the form of (1) small recent haemorrhages. Similar haemorrhages have 
been observed both in blast and contre coup injuries. (2) Areas of softening involving both grey and white matter.

It would appear that (2) represent the result of (1). The only feasible explanations seem to be trauma (direct or blast injury) and multiple small emboli. Owing to lack of history in this case it is not possible to go further. Sections stained by Osmium Tetroxide failed to reveal presence of fat embolism.

\section{(Signed) A W MORGAN Capt RAMC Central Pathology Laboratory, MEF}

\section{Pte G B. 18 Ordnance W/Shops, RAOC}

Evidence of a papular rash was discernible in the trunk and limbs. No area was found which could have been made by an insect bite.

Chest: Heart, right side distended. The myocardium was a pale brown colour and cut like firm cheese.

\section{Lungs: Normal.}

Abdomen: Alimentary tract normal except for 2 small areas of the transverse colon which showed haemorrhagic spots comparable to those on the trunk.

Spleen enlarged to twice normal size.

Supra-renal glands redder than normal, soft and diffluent.

\section{Brain: Normal.}

Conclusion:- In my opinion death was due to Typhus fever.

\section{SGT S W F. 103 MU, RAF}

There was a pronounced icteric tinge of the skin and conjunctivae. This icterus was present in all organs of the body except the brain.

A scar was present on the penis.

Chest: Bile staining of pleurae. Numerous haemorrhages measuring up to $0.6 \mathrm{cms}$ present in the areolar tissue of mediastinum. Sub-pleural haemorrhages. Lungs showed numerous interstitial haemorrhages. Haemorrhage in the heart in relation to coronary vessels. The myocardium was of light colour and did not cut with the usual resilience. The resistance on section resembled nothing as much as cutting a rather ripe banana.

Abdomen: The fatty and areolar tissue of the abdominal cavity all showed haemorrhagic changes, comparable to those seen in mediastinum. The liver was reduced in size. It weighed 1165 grams. In places the capsule had a frosted appearance. The surface beneath the capsule was extensively mottled. On section the tissue tended to bulge beyond the capsule. Large areas of yellow degeneration were present interspersed with smaller darker stained areas. The whole of the normal architecture of the organ was disturbed. No macroscopic evidence of fibrins could be made out. The gall bladder contained no bile.

Further examinations will be made on appropriate tissues to determine the presence or absence of lethal quantities of Arsenic.
Inference: In my opinion death was due to acute yellow命 atrophy of the liver.

Histology: The capsule is wrinkled indicating rapid necrosis of the liver. Complete necrosis of the polygonal cells has? occurred, the cytoplasmic remnants being shrunken and shapeless. Not a healthy polygonal cell is left. There is nof evidence of regeneration, and the necrosis is quite diffuseand not zonal. Round-celled infiltration of the portal tract is observed. (Report from Central Pathologicap Laboratory).

Toxicology: Specimens of liver and hair received fofs estimation of Arsenic content.

The liver contains 1.6 parts per million, equivalent to $1 . \dot{0}$ milligrames of Arsenic as $\mathrm{AS}_{2} \mathrm{O}_{3}$.

The hair contains 2.7 parts per million.

(Report by OC, No 3 Mobile Hygiene Laboratory, MEF) Note: I am indebted to Lt Col R C Menzies, MB, ChB $\stackrel{?}{\omega}$ DPJ, MCRPath, RAMC, OC Leishman Laboratory, Cambridge Military Hospital, Aldershot, for the following statistical figures on Arsenic poisoning.

\section{Normal $0.03 \mathrm{ppm}$ in Liver}

0.3ppm in Hair.
Chronic Poisoning 1-5 ppm Liver (up to $47 \mathrm{ppm}$ )

3 reported fatal cases due to fumes

1.4-4.0 ppm in Liver (mean $2.7 \mathrm{ppm}$ )

\section{ACI J F, RAF. 54 RSO}

Abdomen: Spleen was enlarged to about $2 \frac{1}{2}$ tir normal size. It was dark and congested and firm. The livere only slightly enlarged, and of rather yellow colour.

Brain: Slight excess quantity of pink tinged cerebrospina fluid. The superficial vessels of the brain were congested and the intervening cerebral substance was of pink colour $\frac{9}{3}$ The vessels of the white matter were also congested and prominent and numerous pin point haemorrhages weref present.

Microscopical Preparations. Smears were taken from the spleen and the brain as well as from other organs.

Plasmodium falciparum parasites are present.

Conclusion: In my opinion death was due to infectiono with Malignant Tertian Malaria Parasites. (P. falciparum)

\section{Cpl R L P, ADC, No 8 Mobile Dental Unit}

The extent of the pleural cavities was reduced, by encroachment of the diaphragm. The left pleural cavity was normal, the right contained yellow turbid fluid.

On sectioning the middle and lower lobes of the right lung were compressed and carnified.

A quantity of yellow turbid free fluid was present in the peritoneal cavity. Flakes of lymph were present on the liver and in the paracolic gutters.

The liver was of very much increased size and weighed more than 2000 grams. It was much discoloured. An 
abscess cavity was present in the right lobe. It was immediately subcapsular on the lateral and inferior surfaces and penetrated well into the substance of the lobe. In the more superficial parts the pus that it contained was of semifluid consistency and anchovy paste colour. In the deeper parts it was creamy caseous material. The abscess appeared to be fairly well divided from the liver substance but surrounded by an acute hyperaemia. The greater part of the right lobe showed acute hepatitis and there was gross disorganisation of the liver substance.

Microscopic examination of pus from the hepatic abscess showed the presence of active vegetative forms of Entamoeba histolytica, some containing ingested erythrocytes.

Conclusion: In my opinion death was due to Hepatic Amoebiasis.

\section{Pte M E, 1813 Coy, East African Pioneer Corps}

Large Intestine: The whole of the peritoneal surface of the large gut from caecum to rectum was grossly haemorrhagic, and there were numerous small mesenteric thromboses. On opening the gut the entire length of colon and rectum showed considerable numbers of mucosal ulcers covered wth greyish slough, typical of bacillary dysentery changes.

All coats of the large bowel were thickened, oedematous and haemorrhagic, so much so that in the rectum the lumen was reduced to $1 / 4$ inch diameter.

\section{Conclusion: Death was due to Bacillary Dysentery.}

\section{Pte R H, RASC, EFI}

Adhesions were strong in both pleural cavities - more particularly in the right where the lung could not be dislodged without cutting. Condensations were marked at the right base.

Pyogenic reaction was evident on the left side, particularly in relation to the lower lobe. The lymph was about a centimetre in depth. A pyogenic membrane was present to the left of the mediastinum so placed that the anterior border of the left lung overlay it and it in turn overlay the base and part of the left side of the heart. It was made up of two distinct layers separated by a quantity of yellow liquid under tension. The anterior surface of this structure was smooth and rounded and moulded to the overlying visceral pleura. The posterior surface was closely applied to the subjacent pericardium.

The left lung was consolidated throughout. On section it poured vast quantities of brownish fluid. The right lung showed similar changes in a more advanced degree, in the upper lobe where the pre-existing pneumonia might be reckoned to have gone on to suppuration rather than resolution. The lower lobe of the left lung was collapsed and carnified.

Inference: Death was in my opinion Lobar Pneumonia - Pericarditis - Mediastinitis.

\section{Cpl A K. Free French Forces}

The body was that of a male of negroid appearance $\frac{3}{8}$ There was emaciation of the upper part of the body. Ther was gross swelling of the abdomen and marked oedema of the legs and feet.

Chest: Considerable quantities of clear brown fluid in pleural cavities. Strong pleural adhesions, very tought? adhesion pleura-pericardium. A chain of fibro-caseoug lymphatic glands was seen along left mammary artery; the longest was 1 inch in length. Left lung reduced in size to sized of clenched fist, the tissue was carnified. Right lung was twice the size of left, its lower lobes were carnified and thes only functional lung tissue appeared to be the right upper $\overrightarrow{0}$ lobe. Fibro-caseous pericardium surrounded the heart.

Abdomen: Liver had grossly disturbed architecture ${ }_{\sigma}^{.}$ small area of caseation in relation to vessels. Spleen was] enlarged, dark and firm, and caseous areas were apparent?
in the medulla.

Conclusion: In my opinion death was due to $\overrightarrow{\omega_{0}}$ Tuberculosis-Mediastinitis - Pericarditis Lymphadenitis - Hepatitis.

\section{SECTION III}

\section{Prioriana: Selected Papers on War-time Pathology}

Other documents which are of much interest to us in Defence Forces Medical Services include copies of artictess submitted for publication or I assume intended to submitted. Appropriate extracts from these are quoted below.

\section{TYPHOID FEVER}

Typhoid fever has occurred among the troops in the Middle East in epidemic form mainly in the late summer or Autumn. It appears that the number of cases at any one period has been small. Moreover the clinical appearances of most of these cases is not a little modified by prophylactic inoculations.

The result is that epidemics occur which give quite a little trouble in diagnosis until the right line is struck. The typica features of typhoid fever are present in these but in much less degree than one is accustomed to see them in an' uninoculated population. There is of ten some additiona?. feature which tends to cloud the issue to a greater or lessero extent.

A minor local epidemic of enteric fever occurred recently. The majority of cases were sent to hospital with a provisional diagnosis of malaria as this disease was prevalent at the time. Where certain localising symptom and signs were pronounced provisional diagnosis had been reached of Dysentery, Cerebro-Spinal Fever, Pulmonary Infections, of the latter both Pneumonia and Tuberculosisc were mentioned. 


\section{Case No 3. Pte L. RASC}

This patient felt unwell and complained of headache on Sept 6th. He did not report sick until the 9th Sept and then did so on account of an eczematous condition of both legs.

He was consequently admitted to the Skin Wards of this hospital with a diagnosis of "Infected Eczema of both legs"'!

His temperature on admission was $103^{\circ}$; during the next few days his fever was maintained between $102^{\circ}$ and $104^{\circ}$, headache was severe and he had loose stools.

Blood Films were examined several times but no malarial parasites were found.

On Sept 14th 1942 he was transferred from the Skin Department to the Medical Ward. The fact that he came from the same unit as two other patients admitted to the medical ward who were found to be suffering from Typhoid Fever, coupled with his persistent fever, loose stools and headache led to a strong suspicion of Typhoid.

Blood was taken therefore on Sept 15th for culture and Bact. typhosum isolated.

\section{Investigations}

Among the ancillary methods of diagnosis pride of place must be given to blood culture. In taking a blood culture in abnormal conditions it is important to remember to wash ones' hands after and not before, that is to say one uses a dry and as near as possible " $a$ one finger" technique. By this we mean that the knowledge that one's hands are dirty encourages one to keep them out of the way. One does not touch what one should leave untouched, either the prepared portion of the patient or the business portion of the syringe.

Two other investigations are often carried out in cases of undiagnosed fever with a view to assessing the possibility or the likelihood of an enteric infection; these are the white blood cell count and an Agglutination Reaction.

The character of the WBC count seems to vary from epidemic to epidemic. We have had experience of series of cases which consistently gave counts of up to 15,000 white cells per cu.mm. Even when a leucopenia occurs its value is always difficult to assess. The group of diseases known as Sandfly Fever gives much the same type of count. One can only say that in our experience any epidemic tends in the main to run the same type of count but it is of little help since the conditions one usually wishes to exclude may at the same time be yielding very similar blood pictures.

In military practice one deals with a population that is more than $90 \%$ inoculated. Some may have managed to dodge the column at the last inoculation session but almost every. soldier has had more or less recent experience of typhoid vaccine. In certain of the Services and certain Arms of the service this state of af fairs does not hold to the same degree. However the difficulty of assessing of the probability of these being an enteric group infection from the results of any one agglutination test is colossal.
In effect in routine use agglutination tests are only a
secondary line of attack. In order to achieve anywhere nearo reliable results more than one sample of serum should be examined and even then the evidence may only beo presumptive. It may be necessary to have serum taken in the first, second and third weeks and even when convalescence : is well established.

A further difficulty in assessing the value of agglutinationo tests is the presence of an anamnestic reaction in certaino conditions. This is most marked in cases of exanthematic Typhus. We have observed on occasion where a patient $\stackrel{\square}{\square}$ suffering from Typhus showed a rise in agglutinin titre too $B a c t$ typhosus ' $O$ 'suspension from 30 to 240 in seven days.

\section{Discussion}

Enteric fevers are essentially septicaemic diseases. This is borne out by what is known of their pathology and? particularly of the widespread distribution and $\vec{\omega}$ pleomorphism of complications.

The present series serves to emphasize how widespread are enteric manifestations. That they are in no way confin $\bar{d} d \mathrm{r}$ to the intestinal tract is instanced by the variety of 0 provisional diagnosis in a small epidemic.

In a theatre such as that in which we are at presengitc situated the proportion of undiagnosed fevers labelled? malaria is liable to be high. More than half the present ser were originally thought to be this disease. This rather tends to argue a just appreciation of the clinical aspects of malaria $\biguplus_{\infty}$ since one quickly comes to recognise it as one of the greagt ${ }^{+}$ imitators.

The fact that enteric fever was advanced as a provisional diagnosis in only one case in the present series has rather far $\overrightarrow{ }$ reaching implications. These are first that enteric diseases are of so rare occurrence in civilian practice that the younger $\stackrel{\mathbb{\Omega}}{\complement}$ generation of medical men are unacquainted with it and $\overrightarrow{\vec{F}}$ second that being unacquainted they do not think of it.

\section{BATTLE CASUALTIES POST MORTEM APPEARANCES}

During recent military activity opportunity was found in $\overparen{D}$ this hospital to make as full a post mortem examination as $\frac{\mathscr{N}}{\vec{F}}$ possible on a series of battle casualties. This was done at a time when the hospital was tactically well situated for the 3 . early reception of such cases. During two consecutive $\dot{\delta}$ months seventy three post mortems were done: thirty two of these were battle casualties. Burns cases accounted for 0 seven, twenty were accidental injuries; other surgical conditions yielded four and medical conditions ten.

The hospital at which they were received exercised a glorious impartiality with regard to the origin and type of $\sigma$ case taken. It was conveniently close to Field Medical Units $N$ so that cases could be received which would not have withstood a longer journey. Special facilities also existed $\sigma_{<}$ 
for dealing with maxillo-facial and burns cases. It is thus thought that the series presented will offer a reasonable cross section of the acute type of case which results from modern direct warfare.

In this survey the cases are listed as much as possible under the anatomical site that had aroused the greatest efforts of the clinicians. Where another injury or the extent of complicatory factors were disclosed at post mortem these are indicated. Attempts are made to reconstruct the processes involved clinically.

Skull wounds: Battle casualties coming to autopsy as a result of skull wounds without gross trauma elsewhere were rare. Although all these cases had been afforded surgical treatment in Field Medical Units suppuration had set in and intracranial haemorrhage was the terminal event.

Maxillo-Facial Injuries: These cases provided a few deaths, the majority of these from readily understandable complications. One case who had sustained a wound of the jaw which penetrated to the tongue, succumbed to pleurisy, pneumonia and pericarditis of pneumococcal origin. A second case had sustained a wound in the occipital area where it was well hidden by skin and hair. The missile had travelled through the lateral aspect of the posterior cranial fossa, sufficiently to cause fragments of bone to be pushed into the cerebellum. It had crossed thence to the mouth area, caused a fracture of the lower jaw and done considerable damage within the mouth itself. The cerebellum has reacted normally to the presence of bony spicules in that an abscess cavity was present. Pulmonary oedema had been the terminal event.

Wounds of Limbs: There were two cases of wounding of the limbs which succumbed. One of these was a penetrating wound of the thigh with fracture of the femur. This behaved in a traditional if slightly old fashioned manner by developing and dying of a lobar pneumonia. One case of gas gangrene occurred in the whole period. This patient sustained wounds of the arms and legs. Gangrene developed in both. In spite of radical surgery and local and general specific therapy the infection was overwhelming. Clostridia were isolated from the wounds and stumps as well as from the liver and spleen.

Burns: Seven cases of burns came to autopsy during this period. The problem of the exact mode of death in these cases has been largely debated. In those who do not develop a definite morphological abnormality such as pulmonary oedema, bronchopneumonia, myocarditis or extensive sepsis the principal changes are found in the liver. To these are added certain less well defined and described changes in the duodenum and suprarenals.

It is hoped to add to an already extensive literature by a communication at a later date of which these cases form part of the material.
Wounds of the Abdomen: In this series either the wounding agent or the complications were diverse. On case was wounded close to the hospital and admitted in extremis. Massive transfusions were given and an heroie attempt at surgery involving the removal of the spleen and the left kidney was made. A temporary improvement: resulted but was not sustained. At autopsy a missed rent of the splenic flexure was found. This had resulted in the escape of bowel content. Gross suppuration and도. intraperitoneal haemorrhage were present.

Three cases of abdominal wounding resulting ing suppuration came to autopsy. All had been operated uporn before admission. In two in addition to many and extensive repairs of wounded bowel, a segment had been exteriorised. In both gangrene of the exteriorised segment had set in and $\vec{H}$ been followed by general peritonitis. The third presented a large renal abscess which communicated with the्ष perinephric tissues but drained only a little to the exterior

Wounds of the Thorax and Abdomen: In the presento series deaths from wounds involving the thorax andi abdomen were the most common. Not infrequently the्ष was but one wound present and that a sucking wound of the? thorax. In the cases that came to autopsy it appeared that the general direction of missiles was from abof downwards.

There was but one case which showed abdomirn thoracic type of injury; there was one in which separe wounds had occurred in the abdomen and thorax. In thrgeco cases the thorax alone was wounded.

The one case that showed separate abdominal a thoracic types of injury had received adequate resuscitat treatment, laparotomy and repair, and chest aspiration. At $t$ autopsy bilateral pyothorax was found. Pyogenic reaction was pronounced along the diaphragmatic aspects of the lungs. In the abdomen there were large numbers of adhesions of a great variety of attachments and thicknesses. .3 A large abscess cavity was present in the left upper? quadrant. It was fairly well walled off. It was of irregularo shape and was bounded in front by the posterior aspect of the transverse colon, laterally by the spleen and left kidney. It ran across the surface of the pancreas and then posteriorly so that it then extended up and down anteriors to the transverse processes of the vertebrae. Peritoneafo adhesions had formed just distal to the splenic flexure sof that a condition of subacute intestinal obstruction existed.

The clinical reconstruction of this case appears to be that:the penetrating missiles although not damaging any viscus, carried in infection which proved gross and resistant. Natural reparative processes accounted for the intestinaf obstruction.

The one case that showed abdomino-thoracic injury hade been wounded some days before reaching hospital. Adequate resuscitation had been done. At operation $a$ foreign body had been removed. At post mortem aO subphrenic abscess was found. A track had been ploughed through the right lobe of the liver. This was of somer 
standing and showed a central cavity surrounded by an area of necrotic tissue. This was in turn surrounded by an area of whitish condensed tissue; without this again was an area of hyperaemia. The wounds of the thorax had resulted in pyothorax. At least one loculus showed gas under tension. The thorax was notable for the florid amount of lymph produced and the evidences of infection. In this particular instance the rent in the diaphragm was walled off by the inflammatory reaction.

In this category of autopsies of thoraco-abdominal wounding the changes found, in addition to the trauma, differed first on the amount of haemorrhage and effusion into one or other serous cavity and secondly on the advent of sepsis. The balance has to be struck between those embarrassments caused mechanically and those caused by infection.

In only one case in the present series did the omentum appear to do its duty properly. In this a rent of the diaphragm was firmly and adequately plugged by a tongue of omentum. Abdominal lesions were minimal.

Broncho-pneumonic consolidation in the lung of the unaffected side was one the most common complications. In addition the lung of the unaffected side tended to run with oedema fluid on section.

The natural consequences of penetration of the chest by a missile of course have been haemothorax with greater or less collapse of the lung and displacement of the mediastinum. In nearly every case particularly when there was either a large chest wound or appreciable abdominal damage, infection has been added. In some cases also pneumo-thorax had developed.

The presence of blood in the pleural cavity is a stimulus for the outpouring of pleural fluid. In early cases the fluid found in the pleural cavities is slightly diluted blood. Fibrin thrombi are present on walls in relation to the wounds. In later cases where infection is established the liquid is foul smelling and discoloured. There has been lymph production and pyogenic membranes have formed. Reparative processes are almost always apparent in relation to the track of the missile. In some instances of gross suppuration in the pleural cavity the original track could not be demonstrated.

Evidences of infection within the peritoneal cavity were slight in comparison with those within the thorax. Damage to abdominal organs was not marked in many cases, with the exception of wounds of the liver; it appears that such wounds may be very large without being lethal. In these cases it was the right lobe that suffered. One case of liver wounding had a wound of the kidney also; this was accompanied by peritoneal and thorax haemorrhages and broncho-pneumonia.

Wounds of the lungs themseives seem to have been adequately controlled by the haemorrhage they had occasioned.
A Pathologist Goes to War: The Prior's Papers

Acknowledgements: My thanks are due to Lt Col R K Debenham OBE RAMC for encouragement and permission to use clinical notes. I owe a particular debt to my Laboratory Assistants Sgt Shearman RAMC, Pte J 응 Barrett RAMC, and Pte K Dennis RAMC not only for their of assistance with these post mortems but for their general $\stackrel{\Rightarrow}{\Rightarrow}$ excellence and high morale in this busy time.

\section{BURNS - VISCERAL (HEPATIC) PATHOLOGY}

The older descriptions of the visceral pathology of $\overrightarrow{0}$ burned patients were prodigal not only of the variety of $\overrightarrow{ }$ pictures seen but of theories to account for these changes. $\vec{\omega}$ More recent studies have denied the presence of specific cellular lesion in burns.

This paper gives an account of some of the lesions seen in the livers of burns cases and an attempt is made to correlate them with the therapy given. On this basis it would appear that the actual microscopical change in these livers is a reflection of the agent applied therapeutically. The typ of treatment common to all cases was the administration 을 of large amounts of fluid intravenously. This will obviously. have effect on the amount of congestion seen and o\$ pigment metabolism, and will thus be common in some degree to each section studied.

The livers of thirty-five cases of deaths from burns derived from male adults from twenty to thirty-three years old have been studied. The cases have been divided 1 accordance with local treatment administered:-

Group 1. Cases who received anticoagulants - tannic acio and such like.

Group 2. Cases who received bland treatment principally

saline and saline baths.
Group 3. Cases who received sulphonamides with or without other topical treatment.

The times survived after burning were from instantaneous incineration up to seven hundred hours. More than half the deaths occurred less than one hundred and twenty hours after injury. Of these, half occurred in the first forty-eight hours.

The causes of the burning were airplane crashes, explosions of petrol tanks of cars, primus and petrol cooker accidents, tents catching fire and other forms of accident associated with military service. All were admitted as thermal injuries. No cases of chemical burning as by mustard gas or phosphorus were seen.

A variety of cellular lesions have been described as $D$ resulting from thermal burns; of these the majority of authors place most emphasis of the liver. Central lobular $N$ necrosis was described by Wilson in 1938 as resulting from the burning. Belt in 1939 observed intra-cytoplasmic and intra-nuclear inclusion bodies in the livers of the tannic acid $c$ treated cases. This I was unable to confirm; the technical $\underset{\gamma}{\sigma}$ 
methods were controlled by putting through yellow fever material at the same time. The observations of Erb in 1943 that no central lobular necrosis occurred in the untanned cases is confirmed. The saline treated cases showed only cloudy swelling and some extravasated red blood cells.

The cases treated with sulphonamides were in marked contrast. These showed widespread fatty degeneration; the absence of such change in cases from the other treatment groups who survived longer and were in worse nutritional state tends to exclude the possibility of starvation as a cause of this marked and generalised fatty degeneration.

The possibility of the liberation of a specific substance from a burned surface has been widely canvassed and received experimental support from some authors; in the actual series under review the therapeutic agent gave a profound recognisable effect in the liver.

To summarise, the cellular changes observed in the livers of patients dying from thermal burns could be coupled with the topical therapy.

Those treated with tannic acid showed central lobular necrosis; intra-nuclear and intra-cytoplasmic inclusions were not found.

Those treated with sulphonamides showed fatty degeneration.

No specific changes were found in those who received only bland treatment.

\section{INFLAMMATORY REACTIONS IN PLEURAL FLUIDS RESULTING FROM TRAUMA (Co-author Pte J Barrett, RAMC)}

The reaction of serous membranes to injury has been studied by various workers by injections of suitable doses of bacteria; the serous membrane of choice for experimental study has been the peritoneum of rabbits; there the cellular process is described as first emigration of polymorphs; red cells are present, and this is followed in 18-24 hours by the appearance of large mononuclear cells which are actively phagocytic. These increase in number pari passu with the disappearance of polymorphs. These changes are in turn followed by resolution.

We had the opportunity of studying the cellular reactions in an inflamed serous membrane in human subjects. These injuries were all battle casualties; some of these chest wounds were present together with wounds of the abdomen, The samples taken in each case were obtained at the time of aspiration of the chest; a sample was taken and examined at each aspiration so long as the patients remained in this hospital.

The noxious stimulus in these cases consisted sometimes of a metallic foreign body; to this is added a number of elements derived from the blood stream and occasionally adventitial gas. To these physical stimuli are added bacterial stimuli in a certain proportion of cases.
The reaction to the foreign body was apparently firsk haemorrhage, the clot formation on the damaged pleurae $\frac{3}{\infty}$ and sometimes subsequently infection. This contained and응 possibly infected fluid acted of course as further irritant $\delta$ It is thus thought that the picture presented by the cells of the aspirated fluid gives a picture of the balance between. the organism and the noxious process. That alterations in this fluid do take place in accordance with those proceedingo in the organism as a whole may be inferred from some of the total cell counts when aspiration has been done mores than once.

Particular attention was paid in this study to the presence्ध of cells of the mononuclear series; it was thought that thesemight offer a clue as to prognosis.

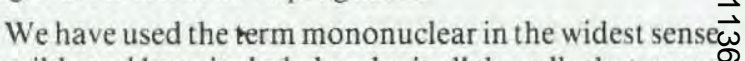
possible and have included under it all the cells that are s@ conveniently described thereby whether they are monocytes of the blood stream, wandering cells from tissue or lymphọ spaces or endothelial cells.

A marked feature of all samples of fluid examined was the absence of clots and platelets. From inspection of togs 5 and of post mortem material it would appear that plateleूs 5 are found, and probably clots formed, only whenı음 haemorrhage is overwhelming.

The reactions observed may be divided roughly into t types (a) those who showed the presence of mononucle ? on first aspiration (b) those who did not.

Of the cases which showed presence of mononuclears first aspiration only one showed growth on culture, sample having large numbers of pus cells, organisms, a number of rather undifferentiated mononuclears.

There were two cases which showed morphological and $\overline{0}$ cultural evidence of infection on first aspiration, on which: occasion also showed no mononuclears, yet which on尺 subsequent examinations showed these cells. In both cases $\overrightarrow{\overrightarrow{0}}$ infection persisted.

We do not think it proper to draw hard and fast conclusions from our small series of pleural fluids aspirated from the chests of wounded cases but we have evidence for 3 . saying that in our experience mononuclears do not tend to occur in the presence of gross infection of pyogenic type In the absence of such infection they are much more readily found.

Acknowledgements: Our thanks are due to Lt Colo Bodley Scott RAMC for the provision of material, clinical?. details and for his general interest.

\section{A CASE OF CEREBRAL CYSTICERCOSIS}

The association of cerebral cysticercosis with epilepsy has ${ }_{0}$ of recent years become fairly weil recognised. Since the publication of MacArthur's paper in 1934 there has everyo 
year been some publication in English journals on the subject and often quite an amount of discussion. The present case is presented with a view to recording one or two minor points of difference from the classical clinical and pathological story.

The patient (25284 Pte Pahlala, J. NMC, UDF attached UDF Camp Reception Hospital, Suez) was an East African native who was admitted to the care of a British Military Hospital suffering from pneumonia. His illness was of one day duration. He was stated always to have been of a lethargic and dull type and unlikely to complain. Within a few hours of his admission he showed generalised twitching of the limbs and rapidly passed into status epilepticus.

No obvious cause could be found for this in examination of blood urea, blood sugar or routine investigations of cerbro-spinal fluid. The urine was within normal limits for a fever. No malarial or other blood parasites were found.

The fits continued without relief and the patient died in a state of coma within 24 hours of his admission.

At post mortem a number of scars were observed on the limbs such as are common in this type of native. On the skin of the trunk was a number of small discrete papular masses the largest of which was some $0.4 \mathrm{cms}$ in diameter. The lungs showed lobar consolidation being at a more advanced stage in the left lung than on the right. There was no other gross abnormality in the viscera of the thorax or abdomen.

Within the cranial cavity there was some slight excess of cerebro spinal fluid. There was some flattening of the convolutions of the right cerebral hemisphere. The superficial vessels showed some congestion. Within the grey matter of the cerebrum and in some cases actually protruding on to the surface was a number of small discrete tumours, each of about $0.4 \mathrm{cms}$ diameter, all of elastic feel and gelatinous texture. Seven of these were present in the cerebrum. They did not cut when the brain substance was incised and they could be extruded from the grey matter by the application of a little pressure.

On finding these tumours a detailed search was made for the indications of the presence of Taenidae but none was found.

Sections of the brain tumours all show larvae of Cysticerus cellulosae. Surrounding the larvae is a condensation of neuroglial tissue but surrounding this was no evidence of regeneration or cellular response. A considerable quantity of fluid was present within the sac so that the embryo was widely separated from the greater part of the wall and could only be seen in relation to the furthest portion of the wall in very low magnifications.

The sections of these cysts present the appearance that have been described in 1941 by Dixon and Willis as 'healthy' or as 'symbiotic'. There is no evidence of disintegration or of calcification or of cellular response of the surrounding cerebral tissue.

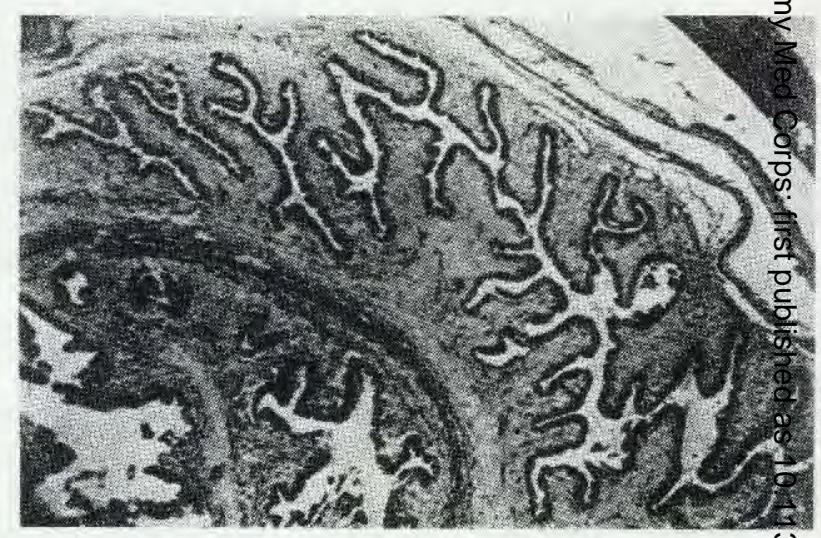

Fig. 2 Oblique section through the scolex showing well $\stackrel{\text { S }}{=}$ preserved 'healthy' larva.

The sections of the tumours and scars of the skin show only scar tissue.

\section{Discussion}

The present case presents certain differences from ti⿱宀 usual story. The patient in this instance required th intercurrence of an acute infective process to bring to ligh cerebral symptoms.

So far as one can gather, in none of MacArthur's cas was this a feature.

The absence of any cutaneous manifestations inclines one to review one's technique, particularly in view of MacArthur's remarks. This has been carefully done but the possibility of having missed a cutaneous or muscular lesion in a post mortem subject must be borne in mind. In 1935 Tull recorded a more or less similar case where no subcutaneous nodules were found but where the brain showed more lesions of the classical type.

In this case probably the most striking feature is the absence of cellular response of the brain and the presence of so called 'healthy' or 'symbiotic' scolices giving rise to symptoms. All the authors consulted agree that the time when the parasite becomes dangerous to the host is when disintegration and calcification set in in the scolex and the cerebral substance shows cellular changes. It would appear that in the present instance the living cysts have under the stimulus probably of an acute systemic disease imbibed fluid making them tense and thus giving rise to symptoms.

\section{Acknowledgements}

My thanks are due to Colonel J S K Boyd DDP MEF for $\tilde{O}$ help and advice and to Captain Kuschlik SAMC for clinical $N$ notes. 


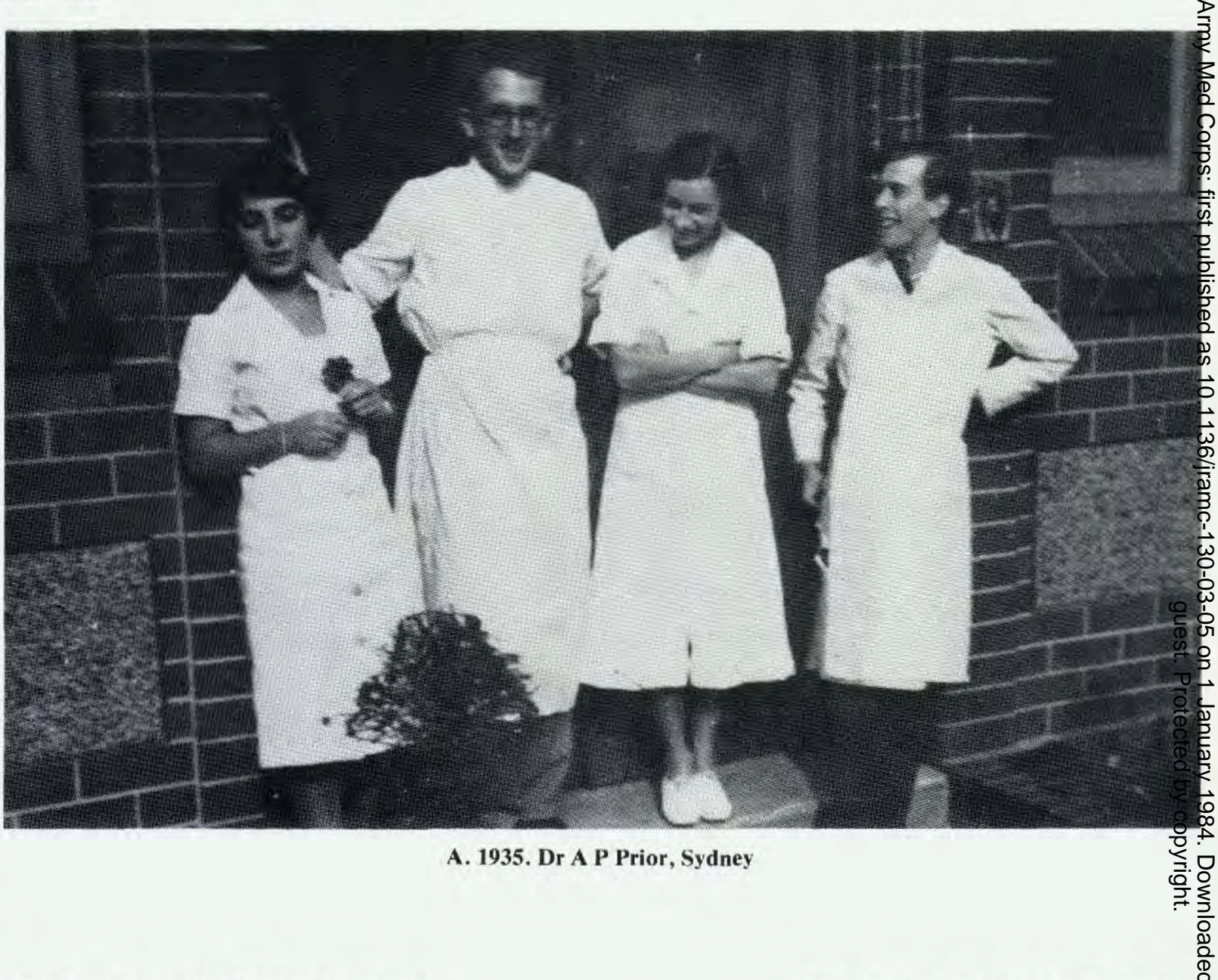

The Transfiguration of Allen Percival Prior

A.

1935 Dr. A P Prior, MB (Sydney)

Resident Pathologist,

North Shore Hospital,

Sydney, Australia.

B. Oct.

1939 P/111781 Lieut A P Prior, RAMC,

MO to 106 Regt, RHA,

MELF.

C. 1942 Lieut to Capt to Major A P Prior, RAMC

Graded Pathologist to Specialist

Pathologist,

8 General Hospital,

Alexandria, MELF.

D. 1943 Pathologist Conference, Cairo.

Who are the other pathologist of ficers in this group photograph?

E. 1945 Major A P Prior, RAMC.

OC 4 Mobile Bacteriology Laboratory,
BLA: Europe (British Army of Liber $\overrightarrow{\vec{A}}$ ation)

F. 12 Jan

1946 Major A P Prior, RAMC (Retd)

G. 1947 Dr A P Prior, MB (Sydney), FRCPath

County Pathologist,

Central Laboratory,

Warwickshire, and later

Pathologist to the South Warwickshire Hospital Group.

From the time of his first paper as a fifth year student there was never a gap of more than two to three yearso without some contribution to medical journals - from post ${ }^{\supset}$ mortem appearances in battle casualties to latent brucellosis in farmers, to ringworm infection in a cucumber greenhouse or lead poisoning from homemade wine $-N$ which is typical of the wide range of interests of that generation of pathologists and of ALLEN PERCIVALN PRIOR in particular. (Lancet, Jan 16, 1971) 


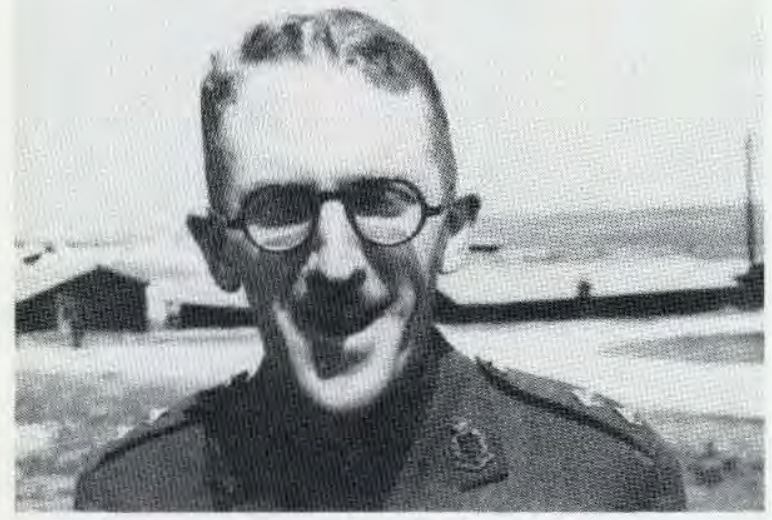

B. Lt A P Prior, RAMC.

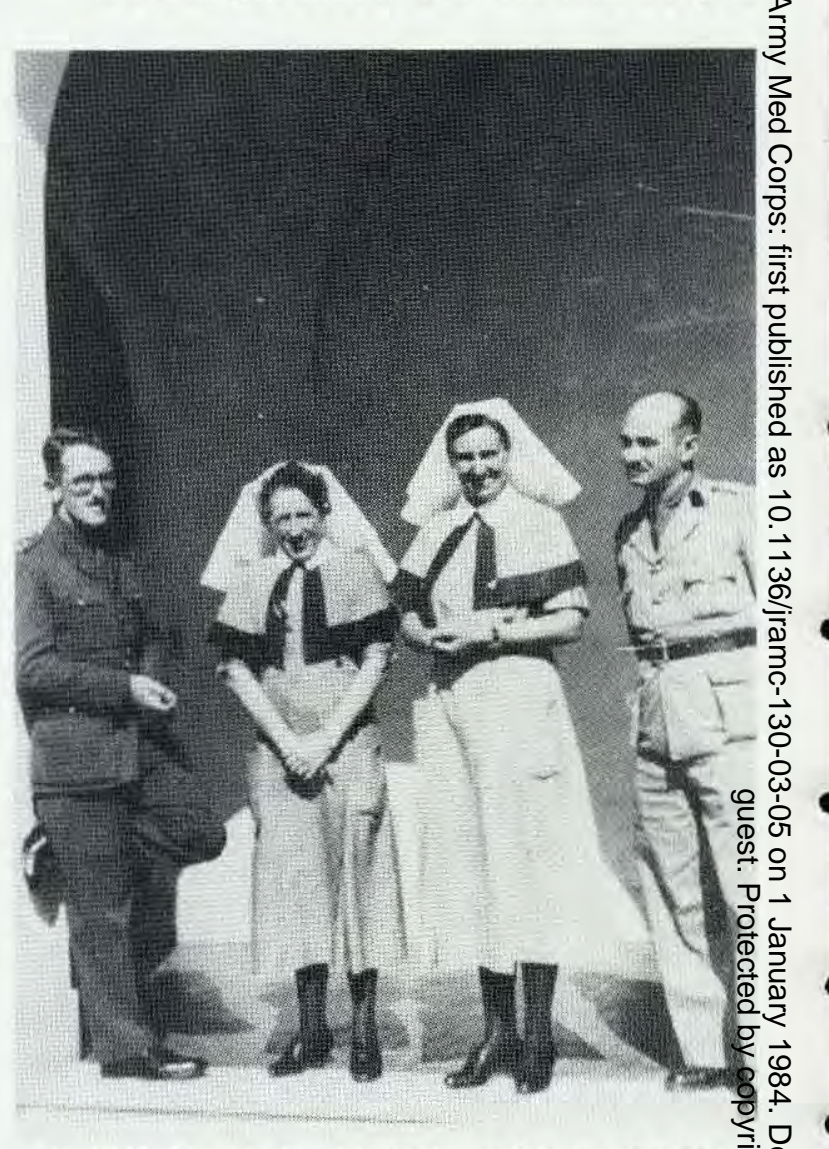

C. 1942. Lieut to Capt to Major A P Prior, RAMC

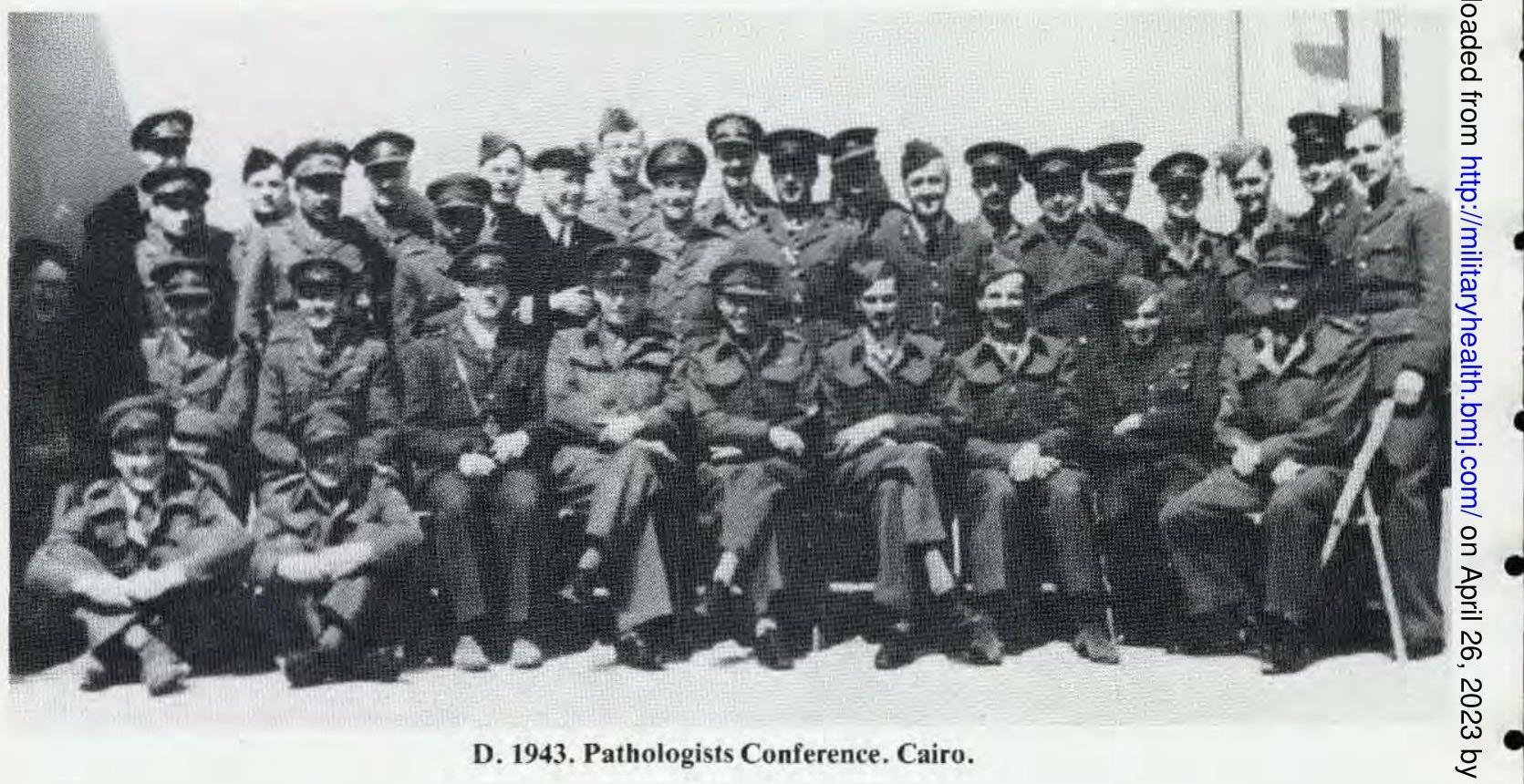




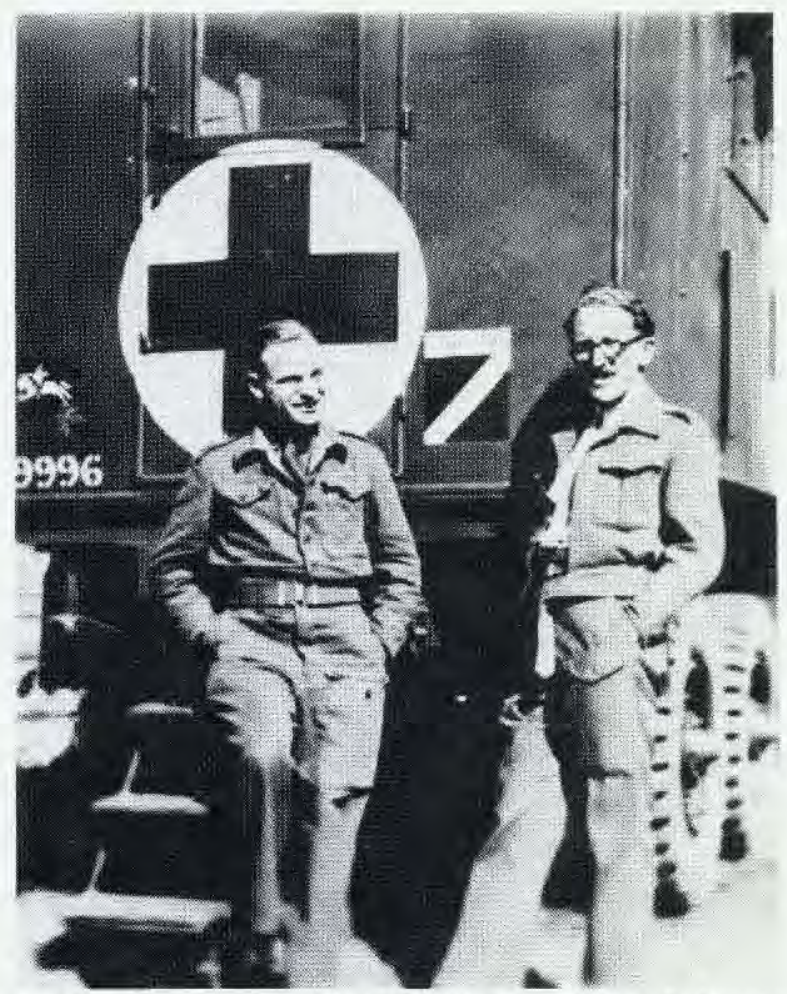

E. 1945. Major Prior, OC 4 Mobile Bacteriology Laboratory
Pf1117:1. (n...5.1)

12th. Janunzy.

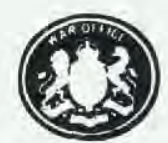

Sir,

Now that the time has cone for your release from active military duty, I am commanded by the Amy Counc: 1 (ृ) to express to you their thanics for the valuable servises which you have rendored in the service of your country $\overrightarrow{0}$ at a time of grave national emergency.

At the end of the cmargency you will relinquist. your comission, and at that time notleioation wil appear in the London Gasette (Bupploment), granting you also the honorary rank of Hajar. Hearshllo, you have parmission to use that rank with effect from the datecof your rolease.

Aajor. an. Prior, (111781)

noyal army edical coms.

c. o. Cot Dionwerlth arik.

ustralin ouse.

trind.

Lontion. 1..2..

एँ F. Major A P Prior, RAMC (Retd), 12 January 1948 an, Sir,

ऽ

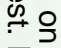
Your obodiont Servant, lesmonter. \%

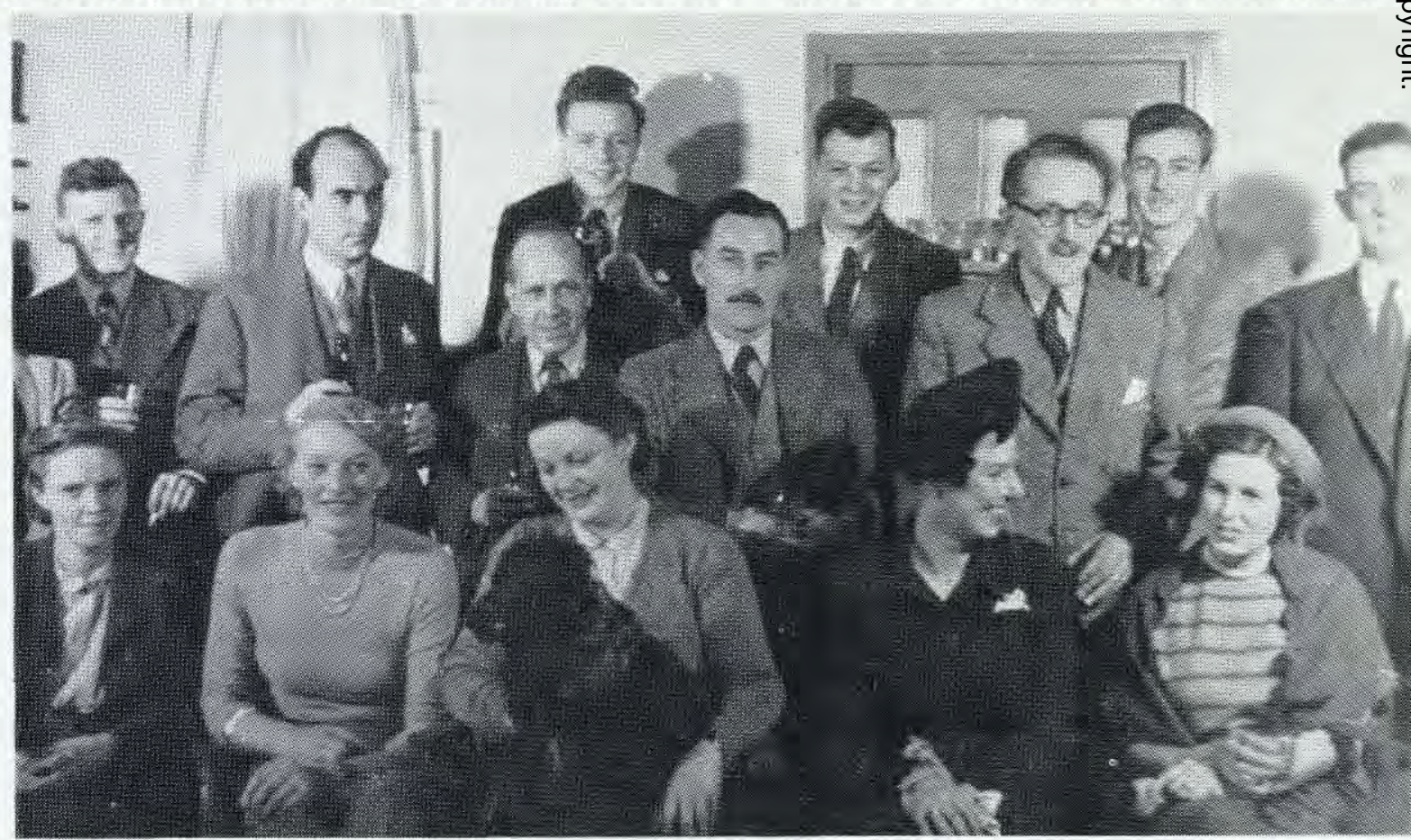

